\title{
A statistical study of the inferred transverse density profile of coronal loop threads observed with SDO/AIA
}

\author{
C. R. Goddard ${ }^{1}$, D. J. Pascoe ${ }^{1}$, S. Anfinogentov ${ }^{1}$, and V. M. Nakariakov ${ }^{1,2}$ \\ ${ }^{1}$ Centre for Fusion, Space and Astrophysics, Department of Physics, University of Warwick, CV4 7AL, UK \\ e-mail: c.r.goddard@warwick.ac.uk \\ 2 School of Space Research, Kyung Hee University, 446-701 Yongin, Gyeonggi, Korea
}

Received 22 April 2017 / Accepted 2 June 2017

\begin{abstract}
Aims. We carry out a statistical study of the inferred coronal loop cross-sectional density profiles using extreme ultraviolet (EUV) imaging data from the Atmospheric Imaging Assembly (AIA) on board the Solar Dynamics Observatory (SDO).

Methods. We analysed 233 coronal loops observed during 2015/2016. We consider three models for the density profile; the step function (model $S$ ), the linear transition region profile (model $L$ ), and a Gaussian profile (model $G$ ). Bayesian inference is used to compare the three corresponding forward modelled intensity profiles for each loop. These are constructed by integrating the square of the density from a cylindrical loop cross-section along the line of sight, assuming an isothermal cross-section, and applying the instrumental point spread function.

Results. Calculating the Bayes factors for comparisons between the models, it was found that in $47 \%$ of cases there is very strong evidence for model $L$ over model $S$ and in $45 \%$ of cases very strong evidence for model $G$ over $S$. Using multiple permutations of the Bayes factor the favoured density profile for each loop was determined for multiple evidence thresholds. There were a similar number of cases where model $L$ or $G$ are favoured, showing evidence for inhomogeneous layers and constantly varying density cross-sections, subject to our assumptions and simplifications.

Conclusions. For sufficiently well resolved loop threads with no visible substructure it has been shown that using Bayesian inference and the observed intensity profile we can distinguish between the proposed density profiles at a given AIA wavelength and spatial resolution. We have found very strong evidence for inhomogeneous layers, with model $L$ being the most general, and a tendency towards thicker or even continuous layers.
\end{abstract}

Key words. magnetohydrodynamics (MHD) - Sun: corona - Sun: UV radiation - Sun: oscillations - methods: observational

\section{Introduction}

The solar corona is highly structured, due to a combination of the low- $\beta$ plasma parameter and the magnetic field that penetrates it from the lower atmosphere. The hot coronal plasma appears to fill in the magnetic flux tubes in certain locations, normally within active regions, forming the curved coronal loops and threads observed by extreme ultraviolet (EUV) imagers. The precise nature of coronal loop formation, and their transverse and longitudinal structure is still debated. The transverse density structure of coronal loops is currently of high importance, as outlined below, and is the focus of this study.

There have been multiple studies of the transverse structure of coronal loops with each generation of EUV imagers (e.g. Bray \& Loughhead 1985; Aschwanden \& Nightingale 2005; Aschwanden \& Boerner 2011; Peter et al. 2013). The majority of such studies note that the transverse intensity profile of the loops resembles a Gaussian peak, which is used to estimate the loop position, width and intensity contrast. To infer the density structure from these intensity profiles the relationship between the density profile of a coronal loop and its appearance in EUV images needs to be understood. The emission in a particular spectral range in the EUV band depends on the plasma density and temperature. Additionally, coronal plasma is optically thin and so multiple structures along the observational line of sight (LOS) will be superimposed in the observations. Finally, the characteristics of the instrument should also be taken into account.
Coronal loops are generally considered to consist of a core of uniform density with an inhomogeneous layer surrounding it. Using data from the Transition Region And Coronal Explorer (TRACE; Handy et al. 1999), Aschwanden et al. (2003) measured the thickness of the non-uniform layer for multiple loops based on a density profile with a sinusoidal transition layer and a uniform core. When the effects of the relationship between the intensity and density, LOS integration and the instrumental point spread function (PSF) were included this density profile was capable of reproducing the observed intensity profiles. Aschwanden et al. (2007) performed a large scale study of the transverse structure of loops, using intensity profiles based on step function and constantly varying density profiles.

Analysed loops have been found to range from near isothermal to highly multi-thermal. Aschwanden \& Boerner (2011) performed a systematic study of the cross-sectional temperature structure of coronal loops using the Atmospheric Imaging Assembly (AIA) on the Solar Dynamics Observatory (SDO; Lemen et al. 2012), finding evidence for near isothermal loop cross-sections. High-resolution Coronal Imager (Hi-C) data was used to measure the Gaussian widths of multiple loops, finding a distribution that peaked at $270 \mathrm{~km}$, the temperature distributions were also found to be narrow (Brooks et al. 2013). Further examples for narrow temperature ranges in coronal loops include (e.g. Warren et al. 2008). However, there are examples of multithermal loops (Schmelz et al. 2010; Nisticò et al. 2014a, 2017) and active, or flaring, loops should also be multi-thermal. 
The unresolved sub-structure of coronal loops is also debated, i.e. loops (or threads) which appear monolithic may be comprised of multiple smaller threads with a certain filling factor. Despite numerous studies using multiple instruments no clear consensus has been reached (e.g. Reale et al. 2011; Brooks et al. 2012, 2016; Peter et al. 2013; Krishna Prasad et al. 2017). However, it appears the lower limit of thread widths is close to being resolved, with a lower limit of $100 \mathrm{~km}$ predicted (Aschwanden \& Peter 2017).

The transverse structure of coronal loops can be determined from, and is integral to, the study of the oscillations they exhibit. Kink, or transverse, oscillations of coronal loops are one of the most intensively studied examples of magnetohydrodynamic (MHD) waves in the solar system. These waves have been clearly observed by EUV imagers such as AIA. The initial detections (Aschwanden et al. 1999; Nakariakov et al. 1999) showed rapid damping, and this is now attributed to resonant absorption (e.g. Ruderman \& Roberts 2002; Goossens et al. 2002). This theory is dependant on the existence of a non-uniform layer, where there is a transition between the density inside and outside the loop. The gradient of the Alfvén speed, and therefore the transverse density profile, then determines the spatial and temporal scales over which the waves are dissipated and the energy is deposited (e.g. Heyvaerts \& Priest 1983; Cally 1991; Soler \& Terradas 2015). The phase mixing length scale defined by Mann et al. (1995) was reproduced by numerical simulations of propagating kink waves (Pascoe et al. 2010), while the corresponding Alfvén wave lifetime has been seismologically calculated using standing kink waves (Pascoe et al. 2016a).

Large scale statistical studies of kink oscillations (Zimovets \& Nakariakov 2015; Goddard et al. 2016) have recently been performed. This work lead to the confirmation of the presence of non-exponential damping envelopes of some of the oscillations studied (Pascoe et al. 2016b). This can be attributed to the damping profile proposed in Pascoe et al. (2012, 2013), which has subsequently been used to perform seismology, including the use of Bayseian model comparison (Pascoe et al. 2016a, 2017a). In Pascoe et al. (2017b) the result of this seismology was compared to density profiles estimated from the EUV intensity for one coronal loop. Arregui et al. (2015) considered the dependence of seismological information on the inhomogeneous layer density profile, without the use of the non-exponential section of the damping envelope, meaning inversion curves were obtained and compared for the different density profiles.

The transverse density structure can also play an important role in understanding and detecting non-linear effects. Terradas et al. (2008) performed a non-linear numerical study of kink oscillations, finding that shear instabilities develop and deform the boundary of the loop. They related their results to the development of the Kelvin-Helmholtz instability (KHI) for torsional Alfvén waves, which was first described by Browning \& Priest (1984). Further numerical studies of the KHI instability for oscillating structures in the corona include; flux tubes (Soler et al. 2010), transverse prominence oscillations (Antolin et al. 2015) and coronal loops (Antolin et al. 2016). In all of these examples the transverse structure is perturbed, which is of theoretical and observational significance. Recently, a study of kink oscillations of coronal loops showed a negative correlation between the quality factor of the oscillations and the amplitude, suggesting the presence of non-linear effects at moderate to large amplitudes, causing real or apparent additional damping (Goddard \& Nakariakov 2016). A similar dependence was found in a numerical study by Magyar \& Van Doorsselaere (2016a), in which non-linear mechanisms such as KHI were found to modify the damping of the kink mode significantly at large amplitudes.

The specific shape of the transverse non-uniformity is also responsible for the geometrical dispersion of the fast magnetoacoustic waves guided by the loop, which determines the specific shape of the quasi-periodic rapidly propagating wave trains (Nakariakov et al. 2004; Mészárosová et al. 2014; Yu et al. 2016). These wave trains have recently been detected in the corona with the EUV imagers (e.g. Liu et al. 2011; Nisticò et al. 2014b), and the full realisation of their seismological potential requires the knowledge of the transverse profile of the waveguiding plasma nonuniformity.

In understanding the mechanisms and effects discussed above, as well as the seismology which is based on them, it is important to understand the transverse and longitudinal loop structure, combining knowledge of the formation and structure of coronal loops and the oscillations they exhibit. In this paper we consider a sufficiently simplified forward modelling procedure which allows us to test which transverse density profile has the most evidence for individual loops based on their observed intensity profile using Bayesian inference. In addition, the structuring parameters with the greatest evidence are obtained for each density model. Our model comparison approach, described in Sect. 3, is the same as that described in Pascoe et al. (2017a).

The paper is organised as follows; in Sect. 2 the observations and data are described, in Sect. 3 the forward modelling and model comparison methods are outlined, in Sect. 4 the results are presented, and the discussion and conclusions are given in Sects. 5 and 6.

\section{Observations}

For this study we are neglecting any time dependent evolution of the loops. For this reason we use single AIA images at 171, 193 and $211 \AA$. One set of images was downloaded for each week between January 2015 and September 2016. Each image was plotted, and loops or threads which appeared monolithic and had a well contrasted segment were identified. This may be an individual thread (or strand), which is part of a larger loop bundle, as long as the width of the thread is sufficient for us to resolved the cross-sectional structure. Two points were selected either side of the loop at a position which minimised background contamination from other structures and maximised the intensity contrast. The intensity was extracted along a line connecting these two points, and was averaged over a width of 5 pixels. The uncertainty and noise on these intensity profiles are considered to be unknown and were inferred during the analysis we describe below. This process resulted in 233 loops for further analysis.

We acknowledge that our sample of loops is not unbiased as loops or threads with a sufficient width to be well resolved and which had no visible sub-structure were selected. Higher, or longer, coronal loops are under sampled, due to the increased noise and reduced intensity contrast making them unsuitable for our analysis.

It was found that the correlation between the loops intensity profile at $171 \AA$ and the other two wavelengths was low in general, implying that the structures studied are not generally multi-thermal over the temperatures sampled by the chosen AIA passbands (which does not exclude them being multi-thermal within a narrower temperature range, or threads with different peak temperatures that are not co-spatial). In general, it did not appear that the intensity profiles at 193 and 211 corresponded to the hotter outer layer counterpart of a cooler core seen in 171 , 
which is often assumed to be the case in forward modelling (e.g. Magyar \& Van Doorsselaere 2016b; Antolin et al. 2016). For this reason we do not extend our analysis to the other wavelengths, and this should be the subject of further study.

\section{Method}

\subsection{Constructed intensity profiles}

In this study we consider three models for the cross-sectional density profile of the coronal loops; the step function profile, the transition layer profile, and the Gaussian profile as described and motivated in Pascoe et al. (2017b). The generalised Epstein profile is not used as the two limits of this profile are well represented by the transition layer profile and the Gaussian profiles. In Pascoe et al. (2017b) it was found that the advantage of the Epstein profile, over the layer profile, as reflected in the Bayes factor, was negligible.

The step function profile (model $S$ ) is described by an internal density $\rho_{0}$, the external density $\rho_{\mathrm{e}}$, and a minor radius $R$. In this case the transverse density profile $\rho(r)$ for a loop with a cylindrically symmetric cross-section and radial coordinate $r$ is given by

$\rho(r)= \begin{cases}A, & |r| \leq R_{S} \\ 0, & |r|>R_{S}\end{cases}$

where $A=\rho_{0}-\rho_{\mathrm{e}}$ is the loop density enhancement.

We also consider a Gaussian density profile (model $G$ ) given by

$\rho(r)=A \exp \left(-\frac{r^{2}}{2 R_{G}^{2}}\right)$.

The linear transition layer profile (model $L$ ) is given by

$\rho(r)=\left\{\begin{array}{crl}A, & |r| \leq r_{1} \\ A\left(1-\frac{r-r_{1}}{r_{2}-r_{1}}\right), r_{1}<|r| \leq r_{2} \\ 0, & |r|>r_{2}\end{array}\right.$

where $r_{1}=R_{L}(1-\epsilon / 2), r_{2}=R_{L}(1+\epsilon / 2)$, and $\epsilon=l / R$ is the transition layer width $l$ normalised to $R$ and defined to be in the range $\epsilon \in[0,2]$. Examples of the three model density profiles are given in the right hand panels of Fig. 1 for three of the analysed loops.

The use of the isothermal approximation allows the intensity profile to be calculated as the square of the density integrated along the LOS. We calculate the loop intensity profile numerically by constructing a $2 \mathrm{D}$ density profile for the radial profiles given in Eqs. (1), (2) and (3) with $r=\sqrt{\left(x-x_{0}\right)^{2}+\left(y-y_{0}\right)^{2}}$, where $x$ is the coordinate transverse to the loop, with the loop centre at $x_{0}$, and $y$ is the coordinate along the LOS.

In addition to the contribution from the loop given by Eqs. (1), (2) and (3), the density profile also includes a background component which is described by a second order polynomial. This is included to model the emission from the background plasma and other structures along the LOS. The instrumental PSF is then applied using a Gaussian kernel with $\sigma=1.019$ pixels, corresponding to the $171 \AA$ SDO/AIA channel (Grigis et al. 2013). The 2D density profile is constructed with 10 times the resolution of the observed intensity profile. The model intensity profiles ( $L, G$ and $S$ ) are then interpolated onto the observational coordinates and compared with the observed intensity profile using the method outlined below.

\subsection{Bayesian inference}

We follow the model comparison procedure based on Bayesian inference and Markov chain Monte Carlo (MCMC) sampling described in Pascoe et al. (2017a) and applied to a coronal loop intensity profile in Pascoe et al. (2017b).

For this procedure priors need to be selected for each of the parameters. An initial least squares fit is performed on the intensity profiles using the forward modelled intensity profile from density profile $L$. This allows guess parameters to be obtained, allowing suitable limits on the priors to be obtained for the $x$ position, radius and intensity contrast of the loop and the background polynomial. For the layer profile we prescribe $0 \leq \epsilon \leq 2$ according to the definition of our density profile. The prior probability distributions of all the above parameters are taken to be constant within the prescribed bounds.

Any two models $M_{i}$ and $M_{j}$ may be quantitatively compared using the Bayes factor, defined as

$B_{i j}=\frac{P\left(D \mid M_{i}\right)}{P\left(D \mid M_{j}\right)}$,

where the evidences, $P(D \mid M)$ are calculated as described in Pascoe et al. (2017a). To define evidence thresholds the natural logarithm of this factor, i.e.

$K_{i j}=2 \ln B_{i j}$,

is considered, where values of $K_{i j}$ greater than 2, 6 and 10 correspond to "positive", "strong", and "very strong" evidence for model $M_{i}$ over model $M_{j}$, respectively. Negative values indicate evidence for model $M_{j}$ subject to the same thresholds. We consider all permutations of the Bayes factor for Models $S, L$ and $G$.

For the purpose of prescribing which model is favoured for each intensity profile, and to what degree, we define the probability of a given model using normalisation of the evidence values as

$P_{i}=\frac{E_{i}}{E_{S}+E_{L}+E_{G}}$,

where $P_{i}$ and $E_{i}$ are the probability and evidence for a given model and $E_{S}, E_{L}$, and $E_{G}$ are the evidence values for models $S$, $L$ and $G$ as described above.

To plot intensity profiles for the models, and plot the distributions of the parameters of interest, we obtain estimates and uncertainties for the model values by taking the median and 95th percentile of the probability distributions for a given parameter.

\section{Results}

\subsection{Model comparisons}

We analysed 233 coronal loops using the method described in Sect. 3, and obtained Bayes factors, $K_{i j}$, and the probability of each model, $P_{i}$, for each loop. Three examples are shown in Fig. 1. The top row shows a loop for which model $L$ was favoured. The corresponding Bayes factors and model probabilities are $K_{L S}=32.3, K_{L G}=26.6, K_{G S}=5.7$ and $P_{L}=1.00$, $P_{G}=0.00, P_{S}=0.00$. On the left is the slit position is plotted in blue, with a close up of the loop inset. The middle panel is the intensity profile (symbols), and model L (blue line), defined using the medians of the returned probability distributions for each parameter. The shaded regions correspond to the $99 \%$ confidence levels for the intensity with and without the modelled noise (red 

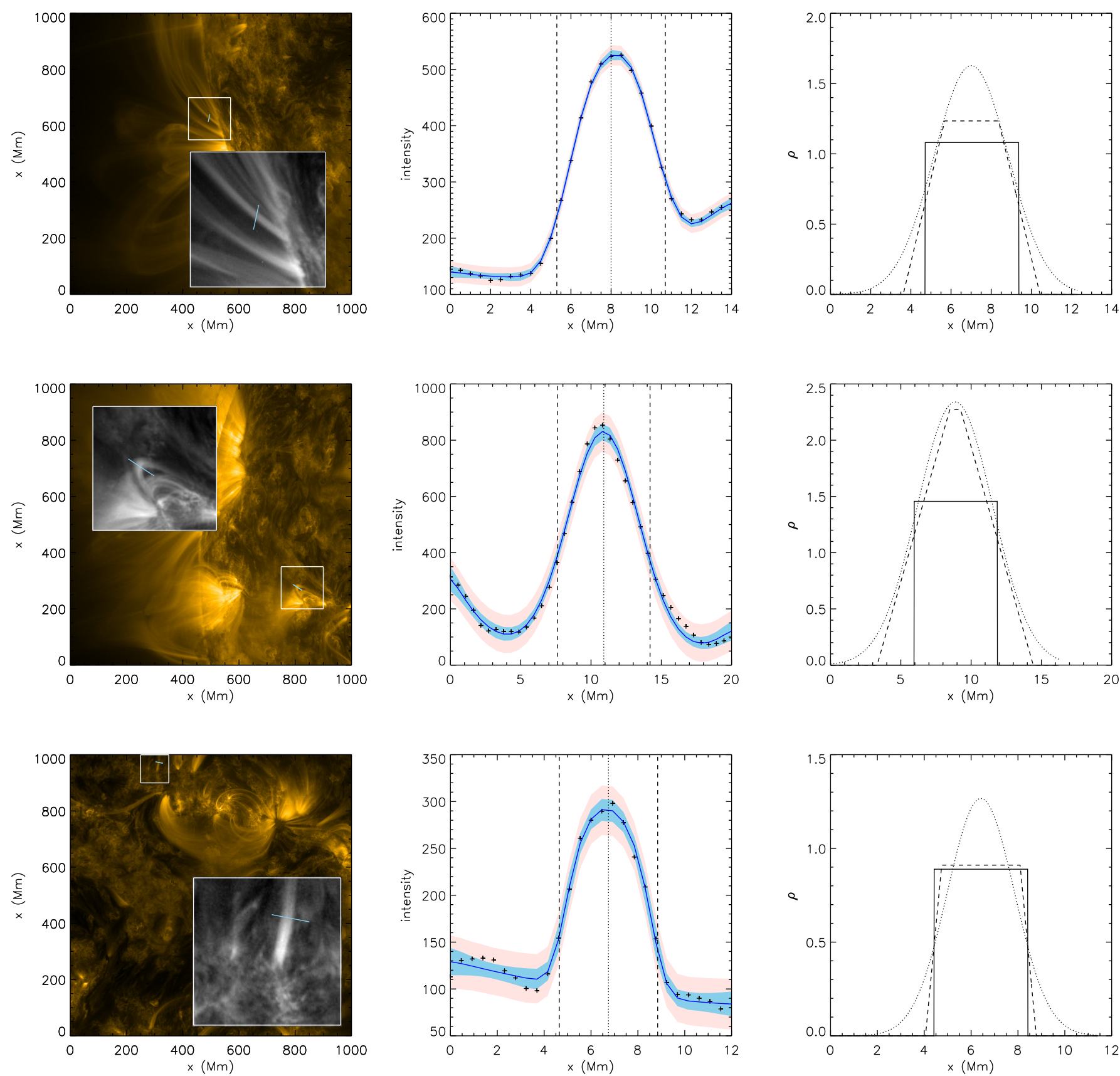

Fig. 1. Examples of loops for which models $L$ (top), $G$ (middle), and $S$ (bottom) were found to best describe the data. Left: SDO/AIA $171 \AA$ Amage of an analysed loop. The blue lines indicates the location of the slits used to generate the transverse intensity profiles. The white box and inset show a magnified region around the loop. Middle: 171 A EUV intensity profile (symbols) across the selected loop. Model $L$ (blue line) is plotted, with the model values being the median values from the corresponding probability distributions. The shaded areas represent the $99 \%$ confidence region for the intensity predicted by the model, with (red) and without (blue) modelled noise. The vertical dotted and dashed lines denote $x_{0}$ and $x_{0} \pm R$, respectively. Right: returned density profiles for models $S$ (solid), $L$ (dashed) and $G$ (dotted).

and blue respectively). On the right the returned density profiles for models $S$ (solid), $L$ (dashed) and $G$ (dotted) are plotted.

The middle row shows a loop for which model $G$ was favoured. The corresponding Bayes factors and model probabilities are $K_{L S}=46.5, K_{L G}=-13.5, K_{G S}=60.0$ and $P_{L}=0.01$, $P_{G}=0.99, P_{S}=0.00$. The bottom row shows a loop for which model $S$ was favoured. The corresponding Bayes factors and model probabilities are $K_{L S}=-2.29, K_{L G}=16.5, K_{G S}=-18.4$ and $P_{L}=0.24, P_{G}=0.00, P_{S}=0.76$. We note that this loop has the smallest radius, and therefore the lowest spatial information.

In Fig. 2 histograms of the Bayes factors $K_{L S}, K_{G S}$ and $K_{L G}$ are plotted. The values of $K_{L S}$ are seen to be largely positive, indicating that Model $L$ is almost always a better model for the density profile of the coronal loops analysed than Model $S$, given our assumptions. The values of $K_{G S}$ are more evenly distributed about zero, indicating that the use of Model $G$ over Model $S$ is not always justified, however there is strong evidence for it in many cases. Finally, the values of $K_{L G}$ are also distributed about zero, with a slight bias to positive values, indicating many loops show strong evidence for either of the profiles over the other.

These results are better quantified by considering the evidence thresholds stated in Sect. 2. These are summarised in Table 1 for $K_{L S}, K_{L G}$ and $K_{G S}$. Each permutation of the Bayes factor is included, with the main result being that in $47 \%$ of cases 

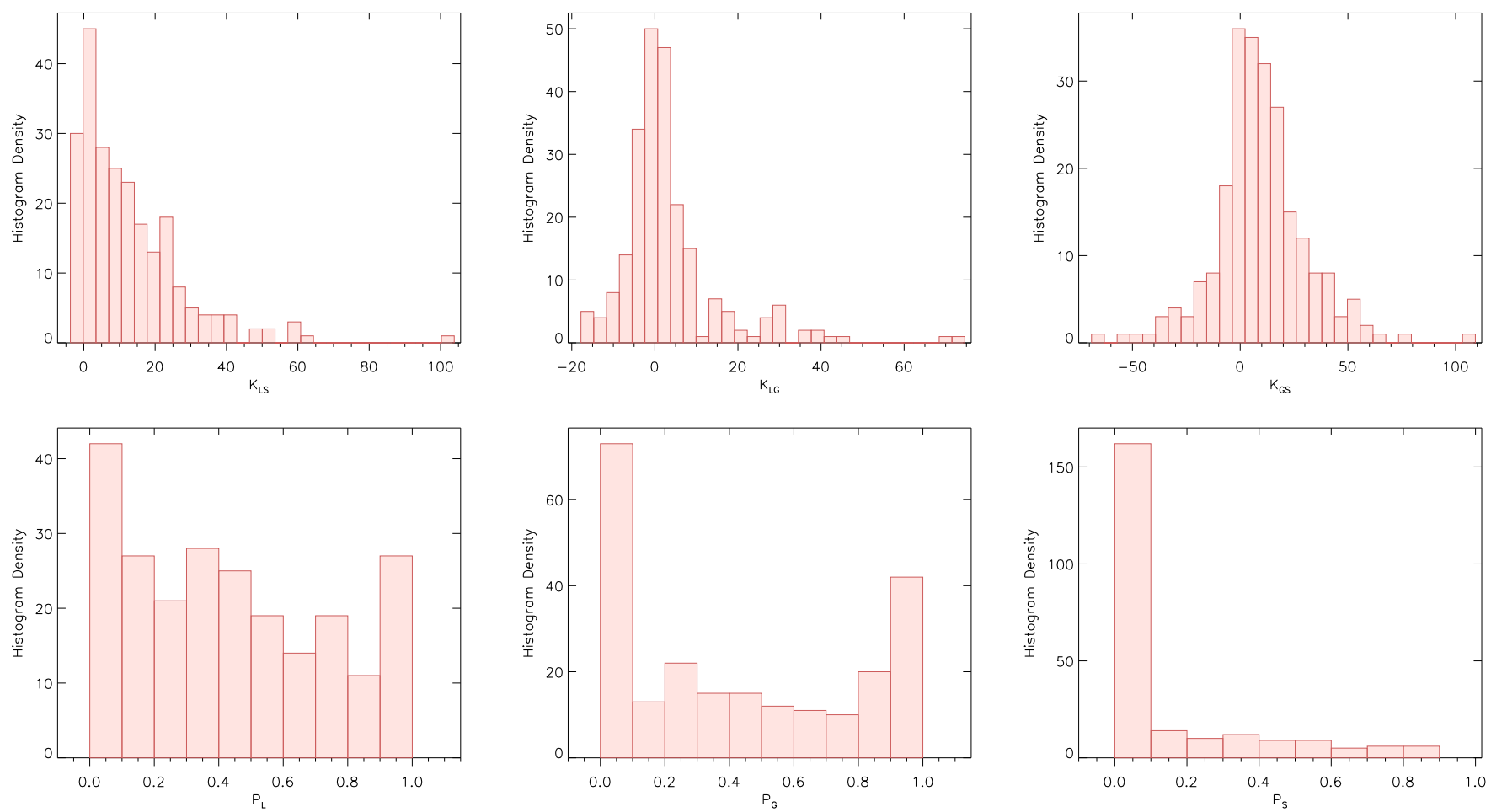

Fig. 2. Top row: histograms of the Bayes factor $\left(K_{i j}\right)$ comparisons of Models $L, G$ and $S$. Bottom row: histograms of the model probabilities $\left(P_{i}\right)$ calculated from the evidence values for each model.

Table 1. Percentages of coronal loop intensity profiles falling into three evidence thresholds for each permutation of the Bayes factor for models $L, G$ and $S$.

\begin{tabular}{llll}
\hline \hline & $K_{i j}>2$ & $K_{i j}>6$ & $K_{i j}>10$ \\
\hline$K_{L S}$ & $75 \%$ & $58 \%$ & $47 \%$ \\
$K_{S L}$ & $4 \%$ & $0 \%$ & $0 \%$ \\
$K_{G S}$ & $65 \%$ & $53 \%$ & $45 \%$ \\
$K_{S G}$ & $25 \%$ & $25 \%$ & $12 \%$ \\
$K_{L G}$ & $42 \%$ & $24 \%$ & $15 \%$ \\
$K_{G L}$ & $32 \%$ & $12 \%$ & $5 \%$ \\
\hline
\end{tabular}

Table 2. A comparison of the evidence for density models $L, G$, and $S$.

\begin{tabular}{llll}
\hline \hline & $L$ & $G$ & $S$ \\
\hline$>0$ & $44 \%$ & $43 \%$ & $13 \%$ \\
$>2$ & $25 \%$ & $32 \%$ & $4 \%$ \\
$>6$ & $8 \%$ & $12 \%$ & $0 \%$ \\
$>10$ & $5 \%$ & $5 \%$ & $0 \%$ \\
$\sum P_{i}$ & 101.5 & 99.4 & 32.1 \\
\hline
\end{tabular}

Notes. Rows 1-4: Percentages of coronal loop intensity profiles falling into four evidence thresholds for each density model. For a loop to be counted for a given model and threshold its Bayes factor from comparison to both other models, $K_{i j}$ and $K_{i k}$, must be greater than that threshold. Row 5: summed probability values $\left(P_{i}\right)$ for each density model, showing how the evidence is distributed between the three models for our 233 analysed loops.

there is very strong evidence for model $L$ over model $S$ and in $45 \%$ of cases very strong evidence for model $G$ over $S$.

These thresholds can be used to determine which of the three models is favoured for each loop, and how strongly. In Table 2 percentages of loops falling into each evidence threshold for each model are listed. For a loop to be counted for a given model $i$, and threshold its Bayes factor for the comparison to the other two models, $B_{i j}$ and $B_{i k}$ must be greater than the threshold. In this case there is a competition between models, so only $5 \%$ of loops have very strong evidence for model $L$ or $G$ over both other respective models.

The probabilities calculated for each model for each loop, $P_{i}$, can be summed to show how the evidence is distributed between the three models. These values are 101.5, 99.4 and 32.1 for model $L, G$ and $S$ respectively, given in Table 2 . This again shows the similarly strong evidence for models $L$ and $G$.

The bottom row of Fig. 2 shows histograms of $P_{L}, P_{G}$ and $P_{S}$. The distributions of $P_{L}$ and $P_{G}$ show a significant number of cases where the respective probabilities are greater than 0.5 , indicating that the corresponding density profile is the favoured model. The distribution of $P_{S}$ shows that the step function density profile was unable to produce the observed intensity profile for the vast majority of cases.

\subsection{Parameter dependencies}

The upper panel of Fig. 3 shows histograms of $\epsilon$ for model $L$ for the different thresholds of $K_{L S}$ and $K_{L G}$ given in Table 2 (red to orange), and with no threshold (grey). These values correspond to the median values from the probability distributions of the parameter. It can be clearly seen that adding the threshold removes the cases where model $G$ was favoured (corresponding to a higher $\epsilon$ for model $L$ ), shifting the distribution to lower values. The cases where model $S$ was favoured are also removed for the higher thresholds, removing the lower values of $\epsilon$. In the lower panel of Fig. $3 \epsilon$ is plotted against the radius for the layer model, $R_{L}$, and shows no correlation. The values of $R_{L}$ also corresponds to the median values of the probability distribution. 

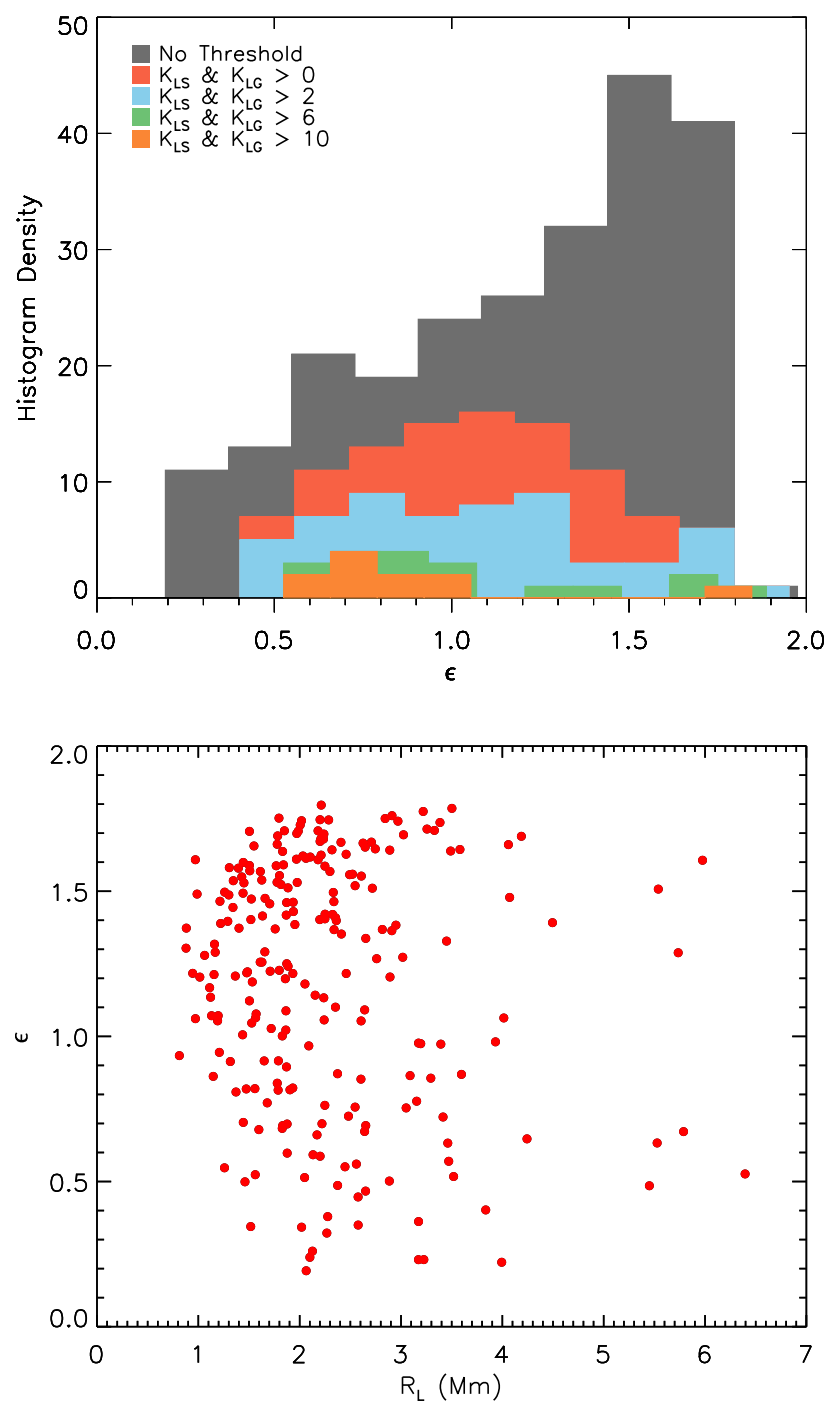

Fig. 3. Upper: histograms of the normalised layer width $\epsilon$ for the combined thresholds of $K_{L S}$ and $K_{L G}$ given in Table 1. Lower: normalised layer width, $\epsilon$, plotted against the loop minor radius for model $L, R_{L}$.

The left hand panel of Fig. 4 shows the distribution of the radii for model $S, R_{S}$. This shows that the sampled loops have radii peaking at $2 \mathrm{Mm}$ with a number of cases with higher radii. The middle panel plots $R_{L}$ against $R_{S}$, where the blue line corresponds to $R_{L}=R_{S}$ and the right panel plots $R_{G}$ against $R_{S}$, where the blue line corresponds to $R_{G}=R_{S}$. This shows that despite the evidence values for the different models varying the radii for the different models remain within error. It can be seen that model $G$ slightly overestimates the radius compared to $S$ and $L$.

In Fig. $5 K_{L S}, K_{G S}$ and $K_{L G}$ are plotted against $R_{L}, R_{G}$ and $R_{L}$ respectively, showing that the spread of Bayes factors increase with loop radius due to the increased spatial information. It can also be noted that $K_{L S}$ is largely positive, whereas $K_{G S}$ is more evenly split between positive and negative values, but with higher values of both. $K_{L G}$ is also more evenly split between positive and negative values, but with the highest evidence values for model $L$ (positive $K_{L G}$ ).

\section{Discussion}

Our results show that in the majority of cases there is evidence for a density profile with an inhomogeneous layer, and in the majority of loops selected there is enough spatial information to constrain the size of the inhomogeneous layer, or note a continually varying profile being preferred. The existence of this inhomogeneous layer between the high density core and lower density background is a necessary and sufficient condition for resonant absorption to occur. It is therefore crucial to the interpretation of transverse loop oscillations in terms of kink oscillations damped by the coupling to Alfvén waves inside the inhomogeneous layer, and hence the validity of any seismological calculations based on this interpretation.

The three cases in Fig. 1 highlight how the different density profiles considered behave for different loops. For the case where model $L$ is favoured model $S$ sets the radius to occur halfway through the inhomogeneous layer and has a correspondingly reduced density contrast. Model $G$ overestimates the width and height of the density profile to match the gradient in the layer of model $L$. For the case where model $G$ is favoured model $L$ reproduces the profile well by minimising the size of the homogeneous core. For the case where model $S$ was favoured model $L$ matches the profile by minimising the size of the inhomogeneous layer. Model $L$ tends to model $S$ in the limit $\epsilon \rightarrow 0$, and so for these cases the additional parameter, i.e. $\epsilon$, is redundant and so model $\mathrm{S}$ is naturally preferred in terms of the Bayesian evidence.

From our results we can see that despite model $G$ being favoured strongly in some cases model $L$ is the most general as it can reproduce both model $S$ and $G$ satisfactorily, while providing additional information where there is evidence for an inhomogeneous layer and homogeneous core. This is encouraging for seismology being performed with model $L$ (Pascoe et al. 2016a), which is the only density profile for which the full analytical solutions are known (Hood et al. 2013) for damping via resonant absorption. However, the many cases in which there is evidence for very large transition layers (or Gaussian density profiles), the thin boundary approximation used would no longer hold. For finite inhomogeneous layers, the damping rate (for the exponential damping regime) is modified by up to $25 \%$ in comparison with the thin boundary approximation (Van Doorsselaere et al. 2004). This may also have implications for the damping and dissipation of the Alfvén waves generated via the resonant absorption of kink fast magnetoacoustic waves. The transverse Alfvén speed profile asociated with the density profile may vary both the energy dissipation rate and it's spatial distribution.

The tables and histograms of the Bayes factors $K_{i j}$ and probabilities $P_{i}$ show that there are a similar number of cases were Model $L$ or $G$ are favoured over the other two, with many extending into the "very strong" evidence threshold. From the bottom panel of Fig. 2, it can be seen that $P_{L}$ is evenly distributed compared to $P_{G}$, which is more confined to low and high values, reflecting the higher generality of model $L$ as discussed above.

From the histogram of $\epsilon$ the distribution without a threshold (grey) shows that the loops analysed generally have large or continuous inhomogeneous layers (where model $G$ was favoured), in contrast to the typically small boundary layers considered in numerical modelling. For the first two thresholds the distribution then centres around $l=R$. In Magyar \& Van Doorsselaere (2016a) it was shown that for thick boundary layers $(\epsilon>0.5)$ there is little or no effect on the exponential damping time at higher amplitudes. However for smaller layers $(\epsilon<0.5)$ the amplitude can have a strong effect on the observed damping time. Our results indicate that loops have inhomogeneous layers which fall on both sides of this threshold, however thicker layers appear to be far more common. 

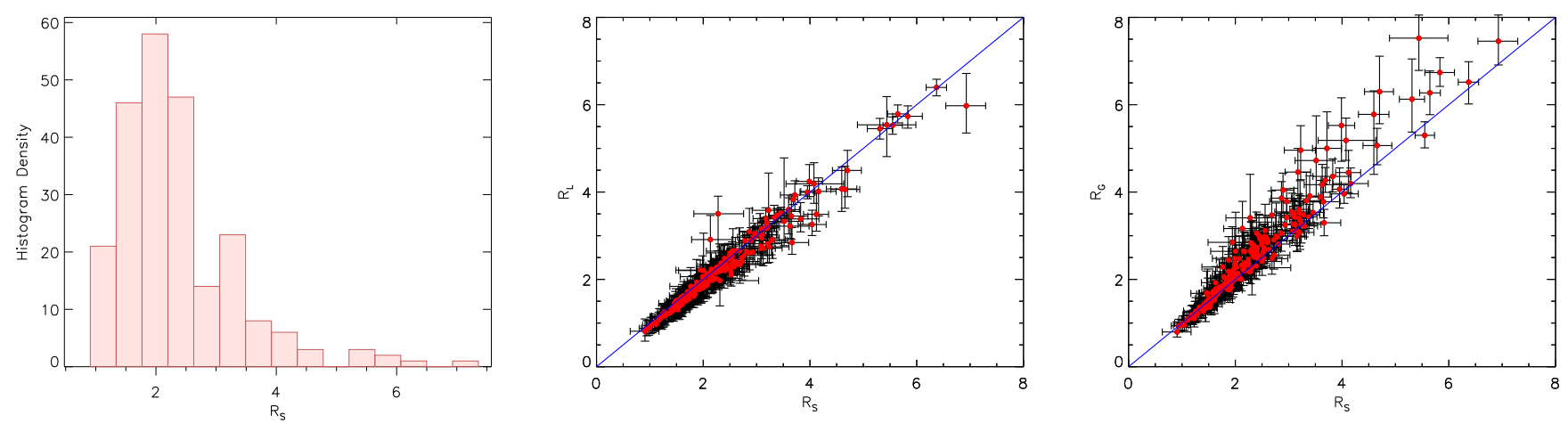

Fig. 4. Comparison of the loop minor radius determined by our three models. Left: distribution of the median radii from model $S, R_{S}$. Middle: loop radii from model $L, R_{L}$, plotted against $R_{S}$. Right: loop radii from model $G, R_{G}$, plotted against $R_{S}$. The blue lines correspond to $R_{L}=R_{S}$ and $R_{G}=R_{S}$ respectively. The error bars correspond to the 95th percentile.
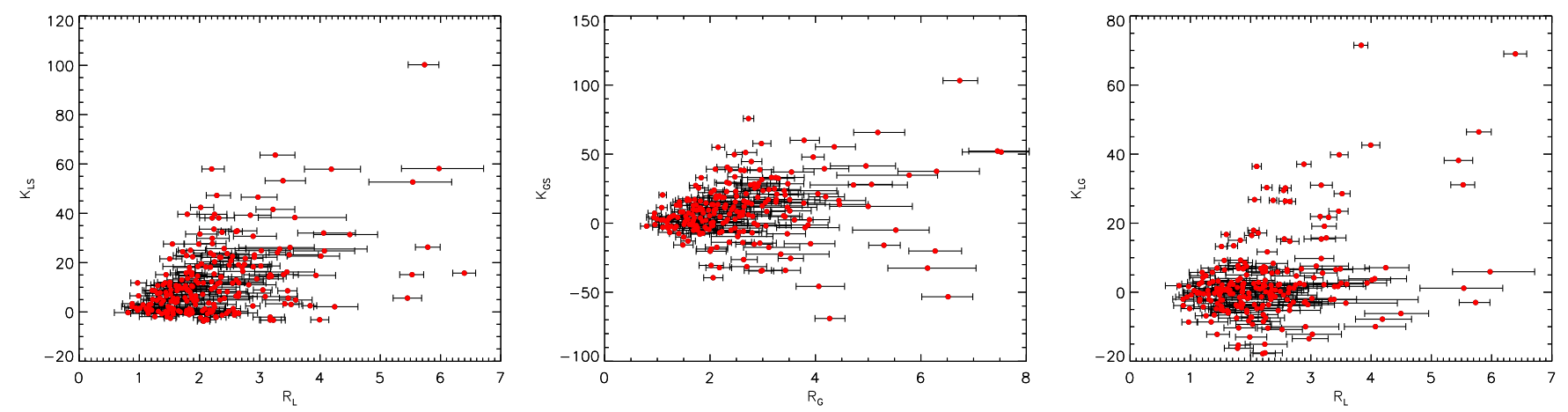

Fig. 5. Left: Bayes factor $K_{L S}$ plotted against loop radius $R_{L}$. Middle: Bayes factor $K_{G S}$ plotted against loop radius $R_{G}$. Right: Bayes factor $K_{L G}$ plotted against loop radius $R_{L}$.

It should be noted that the cases where model $S$ was favoured often corresponded to thinner loops or threads with lower minor radii. This reduction in the spatial information may cause model $S$ to be favoured irrespective of the actual density profile. In some cases the background intensity was not fit well by the second order polynomial, however this is the same for each profile and is reflected in the $99 \%$ confidence levels shown in the middle panels of Fig. 1. It was found that using higher order polynomials for the background trend could lead to different models fitting different portions of the intensity profile, invalidating their comparison.

Our use of the isothermal approximation means that any temperature variation across the loop that is sufficient to vary the response function of the AIA channel analysed will be interpreted as a density variation. This may have contributed to the prevalence of thick or continuous inhomogeneous layers obtained. However, considering the low correlation between the profiles seen in $171 \AA$ and the hotter channels, the structures analysed at $171 \AA$ may have a sufficiently narrow temperature distribution, with separate loops or threads existing in the hotter channels at similar, but not co-spatial, locations.

An additional complication is the potential presence of unresolved substructure in the loops and threads analysed. In this case the density profiles we obtain relate to the density profiles of the unresolved threads, and how the filling factor varies as a function of radius. In the top row of Fig. 1 it is evident that the analysed loop appears to split into multiple structures towards the loop top. This may mean that the intensity profile we have analysed may include several overlapping threads, which affect the transverse structure we infer, however the number of loops analysed is sufficiently large to avoid this being the case for all intensity profiles. Despite these assumptions and complications, in Pascoe et al. (2017b) an agreement was found between the seismologically determined value of $\epsilon$ and the value inferred from the intensity profile. Additionally, numerical simulations of Magyar \& Van Doorsselaere (2016b) suggested that highly multi-threaded, or braided loops could be unstable to transverse oscillations. Since decayless kink oscillations appear to be ubiquitous (Anfinogentov et al. 2015), this indicates that even if resolved loop threads are formed with unresolved substructure, they may quickly evolve to a more monolithic structure.

\section{Conclusions}

In summary, we have analysed the intensity cross-section of coronal loops (and/or threads) observed at $171 \AA$ by SDO/AIA. In this channel typical non-flaring coronal loops are seen with the highest clarity and contrast. Assuming an isothermal and cylindrical cross-section we have inferred the transverse density structure of the coronal loop plasma which lies within the temperature range corresponding to $171 \AA$ SDO/AIA channel.

Accounting for the instrumental PSF and integration along the LOS, very strong evidence was found for the existence of an inhomogeneous layer where the density varies smoothly between the rarified background plasma and the dense centre of the loop. In many cases, the width of this layer was high enough to conclude that the loop does not have a core at all, and has a continuously varying density which may be better modelled by a Gaussian profile. This may have implications for the thin boundary approximation often used in the analytical description 
of oscillating loops. Model $L$ is found to be the most general as it can represent loops with no boundary layer as well as loops with a continuously varying density profile.

We acknowledge that several assumptions have been made to obtain these results. The study of multiple wavelengths, and the inclusion of the instrumental response function and a nonisothermal model for the loop cross-section require further work. The potential presence of unresolved sub-structure, and how this would manifest itself in our observations should also be considered further. We have also assumed the loop is static during the exposure time of the instrument. If they oscillate with a period shorter than the exposure time, or move during the exposure, we would observe some apparent diffusion of its boundary.

From our analysis it is clear that using a linear boundary layer density profile, forward modelled to the resulting intensity profile, produces more information than the Gaussian intensity profiles typically used to fit and track coronal loops. Even with simple least squares fitting, when the spatial resolution is sufficient, this profile would provide information about the size of the inhomogeneous layer compared to the minor radius, and decouples the measured minor radius from the intensity contrast.

Acknowledgements. The work was supported by the European Research Council under the SeismoSun Research Project No. 321141 (C.R.G., D.J.P., S.A., V.M.N.). We thank G. Nisticò for useful discussions which helped seed the initial idea. The data is used courtesy of the SDO/AIA team.

\section{References}

Anfinogentov, S. A., Nakariakov, V. M., \& Nisticò, G. 2015, A\&A, 583, A136 Antolin, P., Okamoto, T. J., De Pontieu, B., et al. 2015, ApJ, 809, 72

Antolin, P., De Moortel, I., Van Doorsselaere, T., \& Yokoyama, T. 2016, ApJ, 830, L22

Arregui, I., Soler, R., \& Asensio Ramos, A. 2015, ApJ, 811, 104

Aschwanden, M. J., \& Boerner, P. 2011, ApJ, 732, 81

Aschwanden, M. J., \& Nightingale, R. W. 2005, ApJ, 633, 499

Aschwanden, M. J., \& Peter, H. 2017, ApJ, 840, 4

Aschwanden, M. J., Fletcher, L., Schrijver, C. J., \& Alexander, D. 1999, ApJ, 520,880

Aschwanden, M. J., Nightingale, R. W., Andries, J., Goossens, M., \& Van Doorsselaere, T. 2003, ApJ, 598, 1375

Aschwanden, M. J., Nightingale, R. W., \& Boerner, P. 2007, ApJ, 656, 577

Bray, R. J., \& Loughhead, R. E. 1985, A\&A, 142, 199

Brooks, D. H., Warren, H. P., \& Ugarte-Urra, I. 2012, ApJ, 755, L33

Brooks, D. H., Warren, H. P., Ugarte-Urra, I., \& Winebarger, A. R. 2013, ApJ, 772, L19

Brooks, D. H., Reep, J. W., \& Warren, H. P. 2016, ApJ, 826, L18
Browning, P. K., \& Priest, E. R. 1984, A\&A, 131, 283

Cally, P. S. 1991, J. Plasma Phys., 45, 453

Goddard, C. R., \& Nakariakov, V. M. 2016, A\&A, 590, L5

Goddard, C. R., Nisticò, G., Nakariakov, V. M., \& Zimovets, I. V. 2016, A\&A, 585, A137

Goossens, M., Andries, J., \& Aschwanden, M. J. 2002, A\&A, 394, L39

Grigis, P., Yingna, S., \& Weber, M. for the AIA team 2013, Tech. Rep., http://hesperia.gsfC.nasa.gov/ssw/sdo/aia/idl/psf/DOC/ psfreport.pdf

Handy, B. N., Acton, L. W., Kankelborg, C. C., et al. 1999, Sol. Phys., 187, 229 Heyvaerts, J., \& Priest, E. R. 1983, A\&A, 117, 220

Hood, A. W., Ruderman, M., Pascoe, D. J., et al. 2013, A\&A, 551, A39

Krishna Prasad, S., Jess, D. B., Klimchuk, J. A., \& Banerjee, D. 2017, ApJ, 834, 103

Lemen, J. R., Title, A. M., Akin, D. J., et al. 2012, Sol. Phys., 275, 17

Liu, W., Title, A. M., Zhao, J., et al. 2011, ApJ, 736, L13

Magyar, N., \& Van Doorsselaere, T. 2016a, A\&A, 595, A81

Magyar, N., \& Van Doorsselaere, T. 2016b, ApJ, 823, 82

Mann, I. R., Wright, A. N., \& Cally, P. S. 1995, J. Geophys. Res., 100, 19441

Mészárosová, H., Karlický, M., Jelínek, P., \& Rybák, J. 2014, ApJ, 788, 44

Nakariakov, V. M., Ofman, L., Deluca, E. E., Roberts, B., \& Davila, J. M. 1999, Science, 285, 862

Nakariakov, V. M., Arber, T. D., Ault, C. E., et al. 2004, MNRAS, 349, 705

Nisticò, G., Anfinogentov, S., \& Nakariakov, V. M. 2014a, A\&A, 570, A84

Nisticò, G., Pascoe, D. J., \& Nakariakov, V. M. 2014b, A\&A, 569, A12

Nisticò, G., Polito, V., Nakariakov, V. M., \& Del Zanna, G. 2017, A\&A, 600, A37

Pascoe, D. J., Wright, A. N., \& De Moortel, I. 2010, ApJ, 711, 990

Pascoe, D. J., Hood, A. W., de Moortel, I., \& Wright, A. N. 2012, A\&A, 539, A37

Pascoe, D. J., Hood, A. W., De Moortel, I., \& Wright, A. N. 2013, A\&A, 551, A40

Pascoe, D. J., Goddard, C. R., Nisticò, G., Anfinogentov, S., \& Nakariakov, V. M. 2016a, A\&A, 589, A136

Pascoe, D. J., Goddard, C. R., Nisticò, G., Anfinogentov, S., \& Nakariakov, V. M. 2016b, A\&A, 585, L6

Pascoe, D. J., Anfinogentov, S., Nisticò, G., Goddard, C. R., \& Nakariakov, V. M. 2017a, A\&A, 600, A78

Pascoe, D. J., Goddard, C. R., Anfinogentov, S., \& Nakariakov, V. M. 2017b, A\&A, 600, L7

Peter, H., Bingert, S., Klimchuk, J. A., et al. 2013, A\&A, 556, A104

Reale, F., Guarrasi, M., Testa, P., et al. 2011, ApJ, 736, L16

Ruderman, M. S., \& Roberts, B. 2002, ApJ, 577, 475

Schmelz, J. T., Kimble, J. A., Jenkins, B. S., et al. 2010, ApJ, 725, L34

Soler, R., \& Terradas, J. 2015, ApJ, 803, 43

Soler, R., Terradas, J., Oliver, R., Ballester, J. L., \& Goossens, M. 2010, ApJ, 712,875

Terradas, J., Andries, J., Goossens, M., et al. 2008, ApJ, 687, L115

Van Doorsselaere, T., Andries, J., Poedts, S., \& Goossens, M. 2004, ApJ, 606, 1223

Warren, H. P., Ugarte-Urra, I., Doschek, G. A., Brooks, D. H., \& Williams, D. R. 2008, ApJ, 686, L131

Yu, H., Li, B., Chen, S.-X., Xiong, M., \& Guo, M.-Z. 2016, ApJ, 833, 51

Zimovets, I. V., \& Nakariakov, V. M. 2015, A\&A, 577, A4 